\title{
Mechanical properties of ceramic-polymer nanocomposites
}

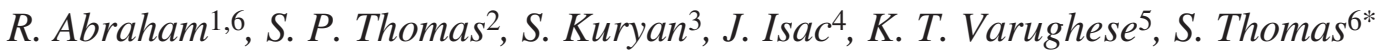 \\ ${ }^{1}$ St Dominics College, Kanjirapally, Kottayam, Kerala-686512, India \\ ${ }^{2}$ Corporate R\&D Division, HLL Lifecare Limited, Trivandrum, Kerala-695012, India \\ ${ }^{3}$ St Stephen's College, Kollam, Kerala, India \\ ${ }^{4}$ CMS College, Kottayam , Kerala, India \\ ${ }^{5}$ Central Power Research Institute, Bangalore, India \\ ${ }^{6}$ School of Chemical Sciences, Mahatma Gandhi University, Kottayam, Kerala-686560, India
}

Received 25 November 2008; accepted in revised form 16 February 2009

\begin{abstract}
Nano crystalline powders of Barium Sodium Niobate (BNN) with the composition $\mathrm{Ba}_{3-2 x} \mathrm{Na}_{4+\mathrm{x}} \mathrm{R} \mathrm{Nb}_{10} \mathrm{O}_{30}$ with ( $\mathrm{R}$ stands for rare earth $=0, x=0$ ) have been prepared by conventional ceramic technique. Barium Sodium Niobate can form a wide range of solid solutions, incorporating rare earth and alkali, alkaline earth elements with different compositions. The powder belonged to tungsten bronze type structure with tetragonal symmetry and lattice constants $a=b=1.2421 \mathrm{~nm}$ and $c=0.3903 \mathrm{~nm}$. XRD (X-ray Diffraction) SEM (Scanning Electron Microscope) and AFM (Atomic Force Microscope) studies revealed that the particle size is in the nanometer range. Composites are prepared by mixing powders of BNN with polystyrene at different volume fractions of the BNN. Melt mixing technique is carried out in a Brabender Plasticoder at a rotor speed of $60 \mathrm{rpm}$ (rotations per minute) for composite preparation. Mechanical properties such as stress-strain behavior, Young's modulus, tensile strength, strain at break etc. are evaluated. Addition of filler enhances the mechanical properties of the polymer such as Young's modulus and tensile strength. The composites showed the trend of perfect adhesion between the filler and the polymer. The filler particles are distributed relatively uniform fashion in all composites and the particles are almost spherical in shape with irregular boundaries. To explore more carefully the degree of interfacial adhesion between the two phases, the results are analyzed by using models featuring adhesion parameter. The experimental results are compared with theoretical predictions.
\end{abstract}

Keywords: nanocomposites, modeling, mechanical properties, adhesion, polystyrene

\section{Introduction}

Materials consisting of more than one kind of substance, that is, composite materials have been designed for complying with various demands. Plastics and rubbers incorporating powdery fillers have frequently been used from the viewpoint of mechanical properties [1]. For these particulate composite materials, it is important to clarify the reinforcement effect of fillers. A variety of theoretical and experimental studies have been reported on the reinforcement effect in terms of macroscopic mechanical properties. However there are a lot of difficulties because of the uncertainty in adhesion between matrix and fillers, dispersion behavior of filler in matrix etc. [2].

In recent years, new approaches are successfully developed for the preparation of polymer composites for electronic applications. Development of electronic devices working at high operating frequencies, such as fast computers, cellular phones, etc. require a new high-dielectric constant materials that combine good dielectric properties with both mechanical strength and ease of processing. The unique combination of dielectric and mechanical 
properties is hard to achieve in a one-component material. Pure polymers are easy to process into mechanically robust components but generally suffer from low dielectric constant [3,4]. On the other hand, typical high-dielectric constant materials, such as ferroelectric ceramics, are brittle and require high-temperature processing [5] which are often not compatible with current circuit integration technologies. The ideal solution would be high-K materials that are mechanically robust and process able at ambient temperatures have to be incorporated with suitable polymers such as ferroelectric ceramic-polymer composites that may combine desired properties of the components $[6,7]$.

An important attribute of polymers is the ability to modify their inherent physical properties by the addition of fillers while retaining their characteristics. Polymers can be made stronger, stiffer, and electronically conductive by the incorporation of various additives. Most of these modifications are made by the addition of inorganic fillers to the polymer. These fillers, present in varying degrees, also affect the basic mechanical properties of the polymer [8-12].

In the present work we have synthesized a new class of ferroelectric ceramic material barium sodium niobate [13-15] and prepared composites out of it by mixing it with polystyrene. In this paper, we report a study on the mechanical properties of polystyrene/barium sodium niobate composites. Tensile modulus, tensile strength and elongation at break data have been analyzed as a function of BNN filler loading on the basis of theoretical predictions for two phase systems. X-ray Diffraction (XRD), Scanning Electron Microscopy (SEM) and Atomic Force Microscope (AFM) studies have been made to characterize the structure and properties of the filler and the composites.

\section{Experimental}

\subsection{Materials}

The filler, ferroelectric ceramic material (Barium Sodium Niobate, BNN) was prepared by usual ceramic technique using reagent grade sodium carbonate, barium carbonate, and niobium pentoxide. Solid-state reaction technique was adopted for the preparation of the material. The starting materials
Table 1. Properties of polystyrene

\begin{tabular}{|l|c|}
\hline \multicolumn{1}{|c|}{ Properties } & Value \\
\hline Dielectric constant & $2.5-2.65$ \\
\hline Poisson's ratio & 0.333 \\
\hline Water absorption & $0.05 \%$ \\
\hline Glass transition temp & $108^{\circ} \mathrm{C}$ \\
\hline Average molecular wt. & 208000 \\
\hline
\end{tabular}

were $\mathrm{BaCO}_{3}$ (Merck Ltd., Mumbai, India), $\mathrm{Na}_{2} \mathrm{CO}_{3}$ and $\mathrm{Nb}_{2} \mathrm{O}_{5}$ (CDH, New Delhi, India). The specific properties of the Polystyrene (Merck Ltd., Mumbai, India) are reported in Table 1.

The constituent carbonates and oxides were weighed according to the chemical formulae satisfying the stoichiometric relations Equation (1):

$$
\begin{aligned}
3 \mathrm{BaCO}_{3}+2 \mathrm{Na}_{2} \mathrm{CO}_{3}+5 \mathrm{Nb}_{2} \mathrm{O}_{5} \rightarrow & \\
& \rightarrow \mathrm{Ba}_{3} \mathrm{Na}_{4} \mathrm{Nb}_{10} \mathrm{O}_{3} \mathrm{O}+5 \mathrm{CO}_{2}
\end{aligned}
$$

The powders were mixed, milled and calcined at a temperature of $1000^{\circ} \mathrm{C}$ for three hours. For the preparation of one gram of $\mathrm{Ba}_{3} \mathrm{Na}_{4} \mathrm{Nb}_{10} \mathrm{O}_{30}$, $0.30948 \mathrm{~g}$ of $\mathrm{BaCO}_{3}, 0.11081 \mathrm{~g}$ of $\mathrm{Na}_{2} \mathrm{CO}_{3}$, and $0.69424 \mathrm{~g}$ of $\mathrm{Nb}_{2} \mathrm{O}_{5}$ were used. To break the agglomerates, which get formed during the calcinations stage, the powder calcined was once again crushed and ground finely. Appropriate quantity of polyvinyl alcohol (binder) was used for proper mixing. Disc shaped pellets (of diameter $10 \mathrm{~mm}$ and thickness 2.5 to $3 \mathrm{~mm}$ ) were prepared at an optimum load of $80 \mathrm{kN}$ and for sintering; the pressed pellets were placed over alumina plate dispersed with zirconium powder. Sintering was carried out at a temperature of $1200^{\circ} \mathrm{C}$ for $5 \mathrm{hrs}$. A heating rate of $10^{\circ} \mathrm{C} / \mathrm{min}$ was allotted before the dwell temperature of $600^{\circ} \mathrm{C}$ and of $50^{\circ} \mathrm{C} / \mathrm{min}$ after the same. The density of the sintered samples was calculated by both sample geometry and Archimedes methods [16]. Then these sintered samples were named as (Barium Sodium Niobate $\left(\mathrm{Ba}_{3} \mathrm{Na}_{4} \mathrm{Nb}_{10} \mathrm{O}_{30}\right)$ or $\left.(\mathrm{BNN})\right)$. The weight $\%$ of the constituent carbonates and oxides were calculated using Equation (1) and presented in Table 2.

Table 2. Composition of BNN

\begin{tabular}{|c|c|}
\hline Material & Content [wt\%] \\
\hline $\mathrm{BaCO}_{3}$ & 27.75 \\
\hline $\mathrm{Na}_{2} \mathrm{CO}_{3}$ & 9.93 \\
\hline $\mathrm{Nb}_{2} \mathrm{O}_{5}$ & 62.31 \\
\hline
\end{tabular}




\subsubsection{Methods of composite preparation}

The melt mixing technique was chosen for preparing the composites because it allowed solvent free mixing for the ceramic filler. By melting at high temperature, molten polystyrene can easily penetrate between filler particles, which facilitates suitable mixing and allow avoiding air trapping into the composites. Consequently void free composites were obtained [17].

Polystyrene-Ceramic composites were prepared in a Brabender Plasticoder. The cavity for mixing in the instrument has an internal volume of $40 \cdot 10^{-6} \mathrm{~m}^{3}$ and is fitted with two screw type rotors of variable speed. Filling the internal cavity completely with mixing charges ensures a constant ram pressure and good mixing. The rotor and the cavity were heated with a circulated flow of electric current and the temperature could be set at any desired level. When the temperature of the internal mixer was raised to $180^{\circ} \mathrm{C}$, polystyrene was added and complete melting of polymer was ensured by a constant minimum torque and attainment of the desired cavity temperature of $180^{\circ} \mathrm{C}$. BNN powder then added to molten PS and was mixed for about 6 minutes at a rotor speed of $60 \mathrm{rpm}$. The compositions of the composites were 10,20, 30 and $40 \%$ by the volume of the filler. The mixed samples were compression molded into sheets of desired thickness by hydraulic press at a temperature of $180^{\circ} \mathrm{C}$ and were used for different studies. The composites were named as BNN10, BNN20, BNN30 and BNN40.

\subsubsection{Measurements}

Energy dispersive X-ray spectrograph was taken by ISIS Link Oxford Instrument, UK. Powder X-ray diffraction data were taken with Bruker X-ray diffractometer using $\mathrm{Cu}-\mathrm{K}_{\alpha}$ line $(0.154 \mathrm{~nm})$ (D8 Advance). The morphology and microstructure of the composites were analyzed by means of high resolution scanning electron microscopy using a JEOL JSM 840-microscope.

Tapping mode Atomic force microscope measurements were carried out in air at ambient conditions $\left(28^{\circ} \mathrm{C}\right)$ with a Veeco Nanoscope $3 \mathrm{D}$, made by Digital Instruments Inc., USA. The characteristics of the measurements are scan rate, scan size and data points. The selected scan rate was $1.001 \mathrm{~Hz}$, scan size $1 \mu \mathrm{m}$ and the image contained 512 data points. Images were analyzed using a Nanoscope image processing software. Particle size is calculated by the particle analysis software 'Nanoscope V531r1. Rectangular samples of $10 \times 1.2 \times 0.2 \mathrm{~cm}^{3}$ were cut for tensile testing. Tensile testing was done using a Universal Testing Machine (Shimadzu, model AG$50 \mathrm{kN}$ ) at a cross head speed of $10 \mathrm{~mm} / \mathrm{min}$. Tensile properties were determined according to ASTM D638.

\section{Results and discussion}

\subsection{Morphological study}

\subsubsection{EDX spectrum}

The EDX spectrum of BNN Figure 1 gives the information on the elemental composition of the material [18]. The $\%$ of carbonates and oxides used for the preparation of $\mathrm{BNN}$ is reported in Table 2. The three dominant peak positions at 1,2.3 and $4.6 \mathrm{keV}$ correspond quite well to the energy pattern of the corresponding materials $(\mathrm{Na}, \mathrm{Nb}$ and $\mathrm{Ba})$ reported in the EDX international chart, giving the evidence that Niobium is dominant in BNN samples. Figure 1 and Table 2 are neatly coincided.

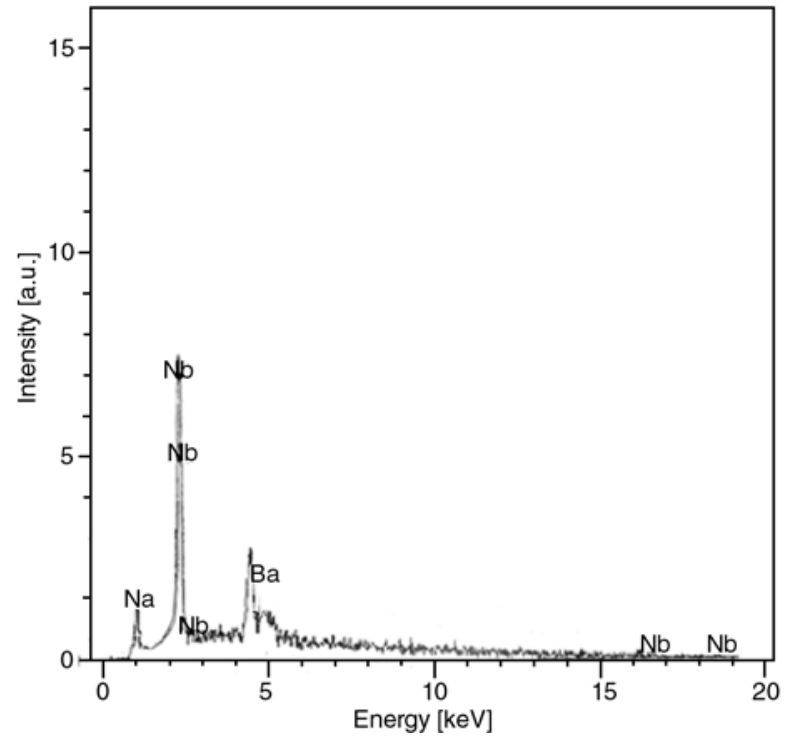

Figure 1. EDX spectrum of BNN

\subsubsection{X-ray analysis of $\mathrm{BNN}$}

Fine powders of BNN were obtained by crushing the sintered pellets. Powder X-ray diffraction pattern of the crushed pellets were recorded in $2 \theta$ range of 10 to $90^{\circ}$ as in Figure 2. Each crystalline solid has its own characteristic X-ray powder pattern, which may be used as a 'fingerprint' for its identification. The lattice parameters of the com- 


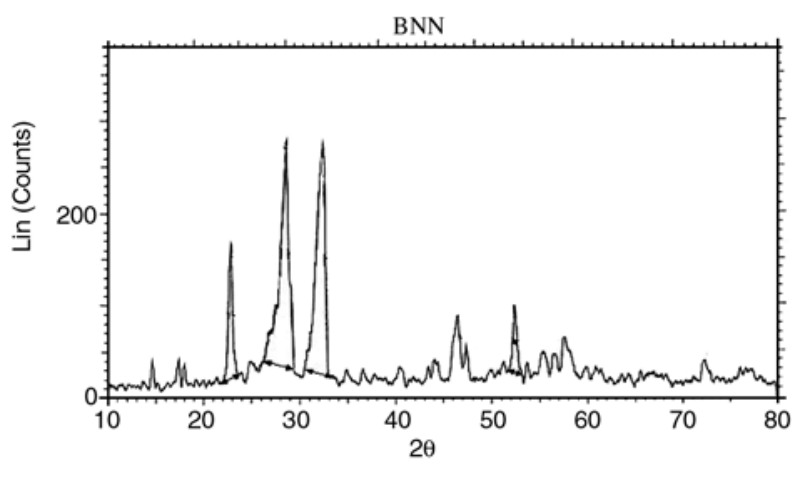

Figure 2. XRD of BNN

pound were obtained using the computer file PDF match to JCPDS data attached to the system analyzer. The patterns were indexed and identified using the JCPDS-ICDD database (Joint Committee on Powder diffraction Standards-International centre for Diffraction Standards-1994). The crystalline phases obtained were structures with tetragonal symmetry. The basic relation for indexing structures with tetragonal symmetry is given by Equation (2):

$$
\frac{1}{d_{h k l}^{2}}=\frac{h^{2}}{a^{2}}+\frac{k^{2}}{b^{2}}+\frac{l^{2}}{c^{2}}
$$

The $d$-spacing of a set of planes is defined as the perpendicular distance between any pair of adjacent planes in the set and it is this $d$ value that appears in Bragg's law. The $d$-spacing of the lines in a powder pattern are governed by the values of unit cell parameters $(a, b, c)$, provided the various lines have been assigned by Miller indices $[h k l]$. The results are again calculated by the computer program PDP1.1 (Powder diffraction Profile 1.1) [19].

All the characteristic peaks of BNN are reported in Table 3. The second and third columns of Table 3 give the intensity count [\%] and intensity [\%] of the diffraction lines. The observed $d$ value from XRD data and calculated $d$ value using the computer program P.D.P 1.1 are reported in the fourth and the fifth column of the Table 3 . The difference between the observed and calculated $d$ value for each reflection is given in the sixth column of the table. From the examination of $\Delta d$ values, it is evident that the observed and calculated values are nearly equal for all major peaks and hence the observed lattice parameters fit the experimental data. So the $d$ value corresponds to the $2 \theta$ value of $22.767^{\circ}$, the [ $h \mathrm{kl}$ ] indices are $\left[\begin{array}{lll}0 & 0 & 1\end{array}\right]$, directly gives the value of $c$-parameter, which is $0.3903 \mathrm{~nm}$ for BNN crystals. The [lllll 002 reflection corresponding to $d$ value of $0.1955 \mathrm{~nm}$ and the $\Delta d$ value 0.0003 . The presence of the above two reflections of the material in the XRD pattern confirm that $\mathrm{BNN}$ belong to tungsten bronze phase with tetragonal symmetry $(a=b \neq c)$. For BNN the lattice parameters of the unit cell axis are $a=1.2421 \mathrm{~nm}$ and $c=0.3903 \mathrm{~nm}$. The axial ratio $(\sqrt{10}(c / a))$ is nearly equal to unity, and volume of the unit cell $602.16 \cdot 10^{-30} \mathrm{~m}^{3}$ which is the characteristics of tungsten bronze type structure with tetragonal symmetry. The space group of the above compound is found to be $\mathrm{P} 4 \mathrm{~mm}$. The $32-$ point groups of crystals can be further classified into $(a)$ crystals having a center of symmetry $(b)$ crystals, which do not posses a centre of symmetry. Crystals with centre of symmetry include 11-point groups and that do not show any polarity. The crystals belong to the remaining 21-point groups do not have a centre of symmetry and posses one or more crystallographically unique directional axes. All crystals in the class of non-centro symmetric point groups show piezoelectric effect. In this group, which shows piezoelectric effect, ten point groups (including 1, 2, m, mm2, 4, 4mm, 3, 3m, 6 and

Table 3. XRD data of BNN

\begin{tabular}{|c|c|c|c|c|c|c|c|c|}
\hline $\begin{array}{c}\text { Angle } \\
(2 \theta)\end{array}$ & $\begin{array}{c}\text { Intensity } \\
\text { count [\%] }\end{array}$ & $\begin{array}{c}\text { Intensity } \\
{[\%]}\end{array}$ & $\begin{array}{c}\text { d value (obs) } \\
{[\mathrm{nm}]}\end{array}$ & $\begin{array}{c}\text { d (cal) } \\
{[\mathrm{nm}]}\end{array}$ & $\Delta d$ & [h k l] & FWHM $\left(\beta^{\circ}\right)$ & $\begin{array}{l}\text { Particle size } \\
\text { [nm] }\end{array}$ \\
\hline 22.767 & 16.00 & 57.8 & 0.3903 & 0.3903 & 0.00 & {$\left[\begin{array}{lll}0 & 0 & 1\end{array}\right]$} & 0.467 & 41.41 \\
\hline 29.298 & 27.5 & 99.1 & 0.3046 & 0.3048 & -0.0002 & {$\left[\begin{array}{lll}4 & 1 & 0\end{array}\right]$} & 0.469 & 39.71 \\
\hline 32.372 & 27.7 & 100.0 & 0.2763 & 0.2768 & 0.0005 & {$\left[\begin{array}{lll}3 & 1 & 1\end{array}\right]$} & 0.503 & 37.72 \\
\hline 46.413 & 8.94 & 32.2 & 0.1955 & 0.1952 & -0.0003 & {$\left[\begin{array}{lll}0 & 0 & 2\end{array}\right]$} & & \\
\hline 47.330 & 5.28 & 19.0 & 0.1919 & 0.1927 & 0.0008 & {$\left[\begin{array}{lll}1 & 0 & 2\end{array}\right]$} & & \\
\hline 52.378 & 9.18 & 33.1 & 0.1745 & 0.1737 & -0.0008 & {$\left[\begin{array}{lll}5 & 4 & 1\end{array}\right]$} & 0.506 & 49.53 \\
\hline 55.358 & 4.81 & 17.3 & 0.1658 & 0.1638 & -0.002 & {$\left[\begin{array}{lll}4 & 1 & 2\end{array}\right]$} & & \\
\hline 56.456 & 4.7 & 16.9 & 0.1628 & 0.1624 & -0.0004 & {$\left[\begin{array}{lll}3 & 3 & 2\end{array}\right]$} & & \\
\hline 57.565 & 6.57 & 23.7 & 0.1600 & 0.1601 & 0.0001 & {$\left[\begin{array}{lll}7 & 1 & 1\end{array}\right]$} & & \\
\hline 72.318 & 4.29 & 15.5 & 0.1306 & 0.1306 & 0.0000 & {$\left[\begin{array}{lll}7 & 1 & 2\end{array}\right]$} & & \\
\hline
\end{tabular}


$6 \mathrm{~mm}$ ) have only one unique direction axis. Such crystals are polar crystals and they show spontaneous polarization and ferroelectric effect [20].

The particle size is calculated from X-ray diffraction profiles of strong reflections with intensity $\%$ greater than 30 using Scherrer's equation by measuring the full width at half maximum (FWHM). The (FWHM) of the material is reported in the eighth column of Table 3 and the calculated particle size is in the last column of Table 3. The Debye Scherrer equation (3) for calculating the particle size is given by Equation (3) [21]:

$$
L=\left(\frac{0.9 \lambda}{\beta \cos \theta}\right)
$$

where $\lambda$ is the wavelength of light used for the diffraction, $\left(\mathrm{Cu}-\mathrm{K}_{\alpha}\right.$ line) $(0.154 \mathrm{~nm})$ and $\beta$ is the 'full width at half maximum' of the sharp peaks, and $\theta$ is the angle measured from the data chart. The calculated values of lattice parameters for BNN, which are in close agreement with values reported in the literature [22]. X-ray powder diffraction may be used to measure the average crystal size in a powdered sample, provided the average diameter is less than about $200 \mathrm{~nm}$. The lines in the powder diffraction are of finite breadth but if the particles are very small the lines are broader than usual. The broadening increases with decreasing particle size. The limit is reached with particle diameters in the range roughly 2 to $10 \mathrm{~nm}$; then the lines are so broad that they effectively 'disappear' into the background radiation.

The results reveal that the particle size of $\mathrm{BNN}$ is less than $100 \mathrm{~nm}$. Since XRD is a first hand measuring technique for particle size calculations, one can prefer measurement using SEM or AFM for particle characterization.

\subsubsection{X-ray analysis of the composites}

The particle size of the ceramic powder and various composites are calculated from X-ray line broadening studies using Scherrer's equation. Figure 3 is the XRD pattern of PS. The particle sizes in all compositions are observed as almost same and reveal the powder is well dispersed in PS medium. Diffraction data is collectively given in Figure 4. All the peaks of the composites are identified and the $[h k l]$ values are indexed. Humps seen in the

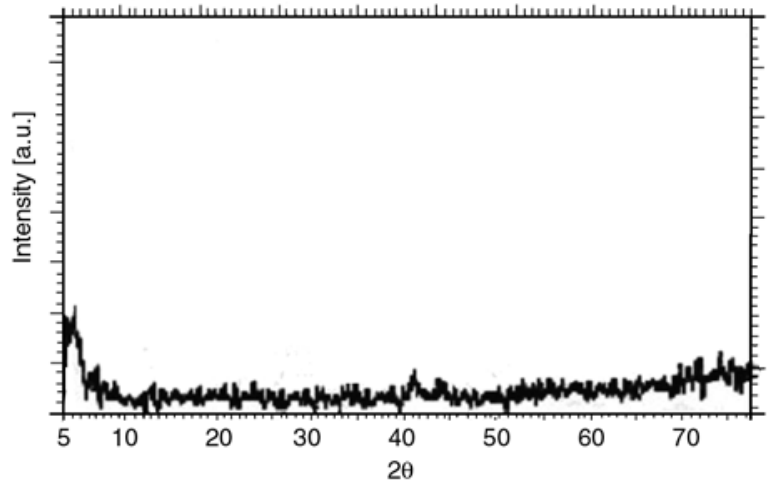

Figure 3. X-ray Diffraction pattern of Polystyrene

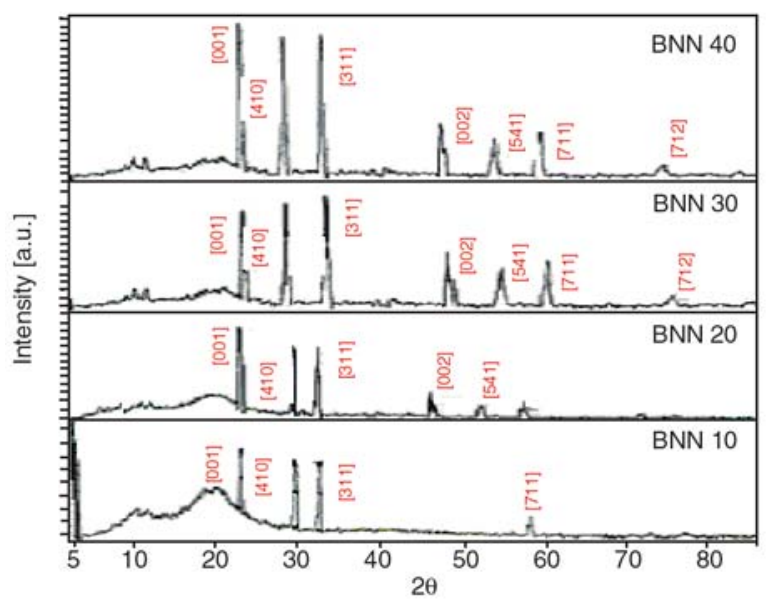

Figure 4. X-ray Diffraction pattern of Composites

left side in the diffractogram Figure 4 up to a $2 \theta$ of $20^{\circ}$ characterize the amorphous nature of PS and they gradually diminish with the increment of filler content. The emergence of characteristic diffraction lines and their gradual sharpening increases with filler content and for BNN40 all most all the characteristic peaks of BNN are present. Strong and sharp three peaks of intensity $57 \%$ at $22.767^{\circ}$ whose [ $h \mathrm{kl} l]$ values [ $\left[\begin{array}{lll}0 & 0 & 1\end{array}\right]$ of intensity $99.1 \%$ at $29.298^{\circ}$ whose $[h k l]$ values at $\left[\begin{array}{lll}4 & 1 & 0\end{array}\right]$ and of intensity $100 \%$ at $32.372^{\circ}$ whose [ $\left.h k l\right]$ values at $\left[\begin{array}{lll}3 & 1 & 1\end{array}\right]$ of BNN are seen clearly in all BNN-PS composites. Composites also showed an XRD pattern in which the basic peaks of BNN are intact in the composite, (the peak position didn't change and the particle size remains the same as the parent material) indicating that the parent material does not undergo any transformation during the preparation of the composite. Thus the comparison of XRD patterns of PS and the composites confirmed that BNN does not react with PS. The lattice parameters of the constituent phases are almost the same in all composites. This indicates that the structure remains the 
same even if the composition of composites is varied. No other additional peak is observed apart from the parent material. All the above evidences confirmed the successful preparation of the two phase composite material This sequence is generally consistent with the study of Panajkar et al. [23] and the recent work of Lee et al. [24] who detected the unchangability of XRD peaks of the fillers by composite formation. Intensities of $\mathrm{X}$-ray reflections are important for two main reasons. First, quantitative measurement of intensity is necessary in order to determine unknown crystal structures. Second, qualitative intensity data are needed to characterize materials and identify unknowns. The relative measure of the crystalline phases of the BNN composites can be obtained from the intensity of the scattered beam. From the Figure 4 it is concluded that with respect to the increment of filler content the magnitude of intensity increases and that reveal the increment of crystalline particles in the composites.

\subsubsection{AFM images of the composites}

AFM topographic images of the surfaces and cross sections of the composites are used for this study. In Figure 5a, 5b) the cross sectional images of selected composites are given. Images show some coloured areas in the images. These are areas where
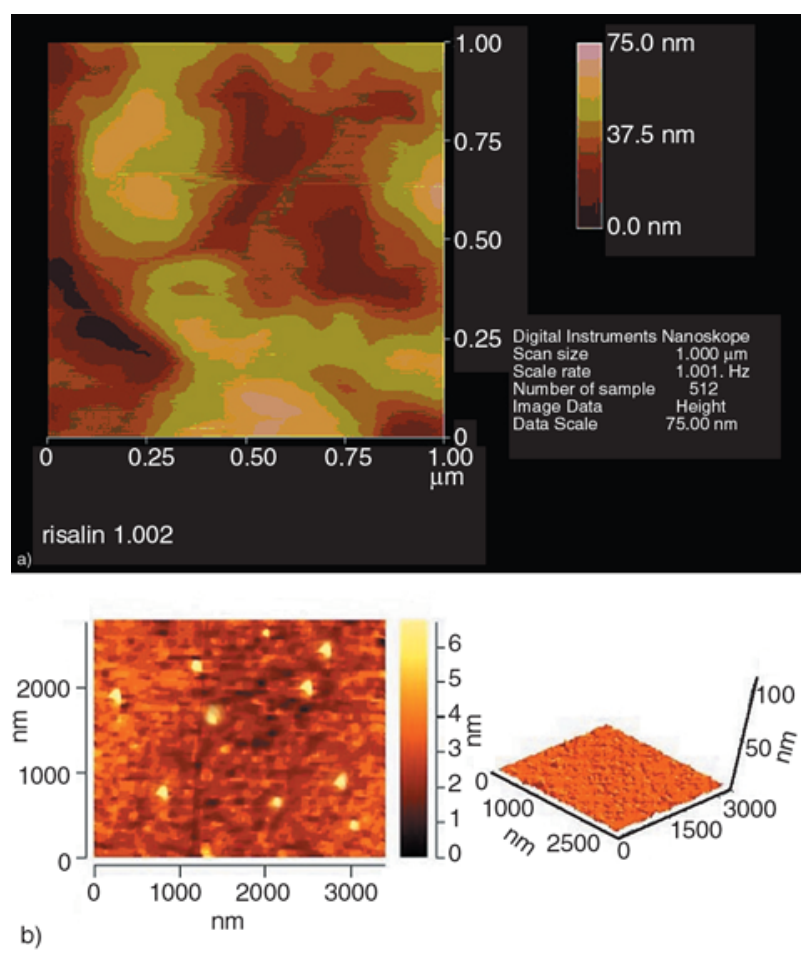

Figure 5. AFM image of BNN10 (a) and BNN40 (b)
Table 4. Particle size measurement through software

\begin{tabular}{|l|c|c|}
\hline \multicolumn{1}{|c|}{ Dimension } & Mean & Minimum \\
\hline Height & $2.58 \mathrm{~nm}$ & $-0.91 \mathrm{~nm}$ \\
\hline Area & $10059 \mathrm{~nm}^{2}$ & $15.259 \mathrm{~nm}^{2}$ \\
\hline Diameter & $58.14 \mathrm{~nm}$ & $4.40 \mathrm{~nm}$ \\
\hline Length & $119.38 \mathrm{~nm}$ & $5.52 \mathrm{~nm}$ \\
\hline Width & $38.29 \mathrm{~nm}$ & $5.52 \mathrm{~nm}$ \\
\hline
\end{tabular}

the particles are located in the PS matrix. While increasing the filler amount the number of such spots increases indicating the dispersion of the fillers in the matrix. By the AFM image Figure 5a we get a particle size of the order of $75 \mathrm{~nm}$ (rose coloured) for BNN from the image of the filled samples. The average particle size calculated by the software (Nanoscope particle analyzer V531r1) is reported in Table 4 and its value is $58 \mathrm{~nm}$.

\subsubsection{SEM Analysis}

Morphology has been analyzed from Scanning Electron Microscope. The average particle diameter is found to be less than $100 \mathrm{~nm}$ in all composites. Three pairs of images of BNN10 and BNN40 with different magnification are given in Figure $6 \mathrm{a}$ and $6 b$, Figure $7 a$ and $7 b$ ), and Figure $8 a$ and $8 b$. The ceramic particles appear to be well dispersed in both low and high concentration composites. Clustering or agglomeration is seen to be absent in all BNN-PS composites. The filler particles are distributed relatively uniform fashion and the particles are almost spherical in shape with irregular boundaries. The filler particles are seen to be clearly embedded in the polymer matrix, which establishes the (0-3) connectivity of the composites. The ceramic-polymer composites are made up of an active ceramic phase embedded in a passive polymer phase. The properties of the composite depend on the connectivity of the phases, volume percent of ceramic, and the spatial distribution of the active phase in passive phase. The concept of connectivity developed by Newnham [25] describes the arrangement of the component phases within a composite. It is critical in determining the electromechanical properties of the composite. There are 10 different types of connectivities possible in a diphasic composite. It is shown in the form $(A-B)$ where $A$ refers to the number of directions in which the active phase is self connected or continuous. $B$ shows the continuity directions of the passive phase. In BNN- 


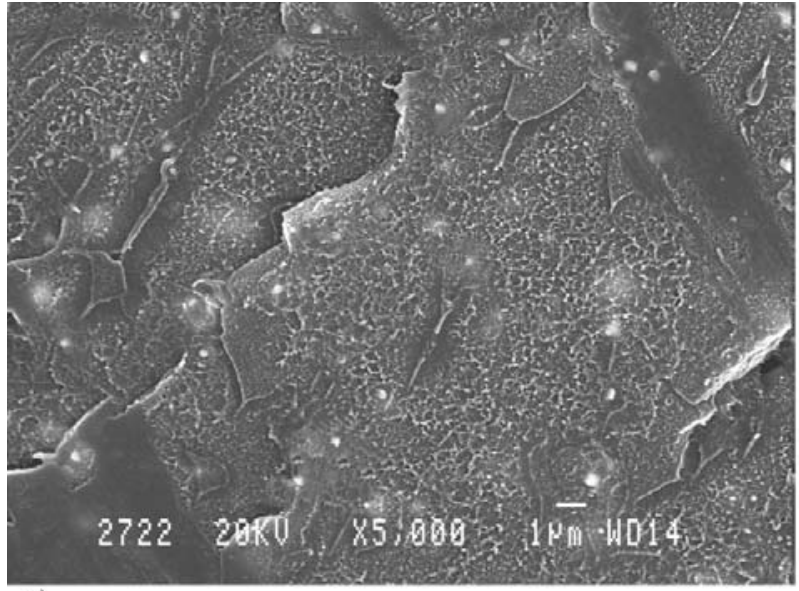

a)

Figure 6. Fracture surface image of BNN10 (a) and BNN40
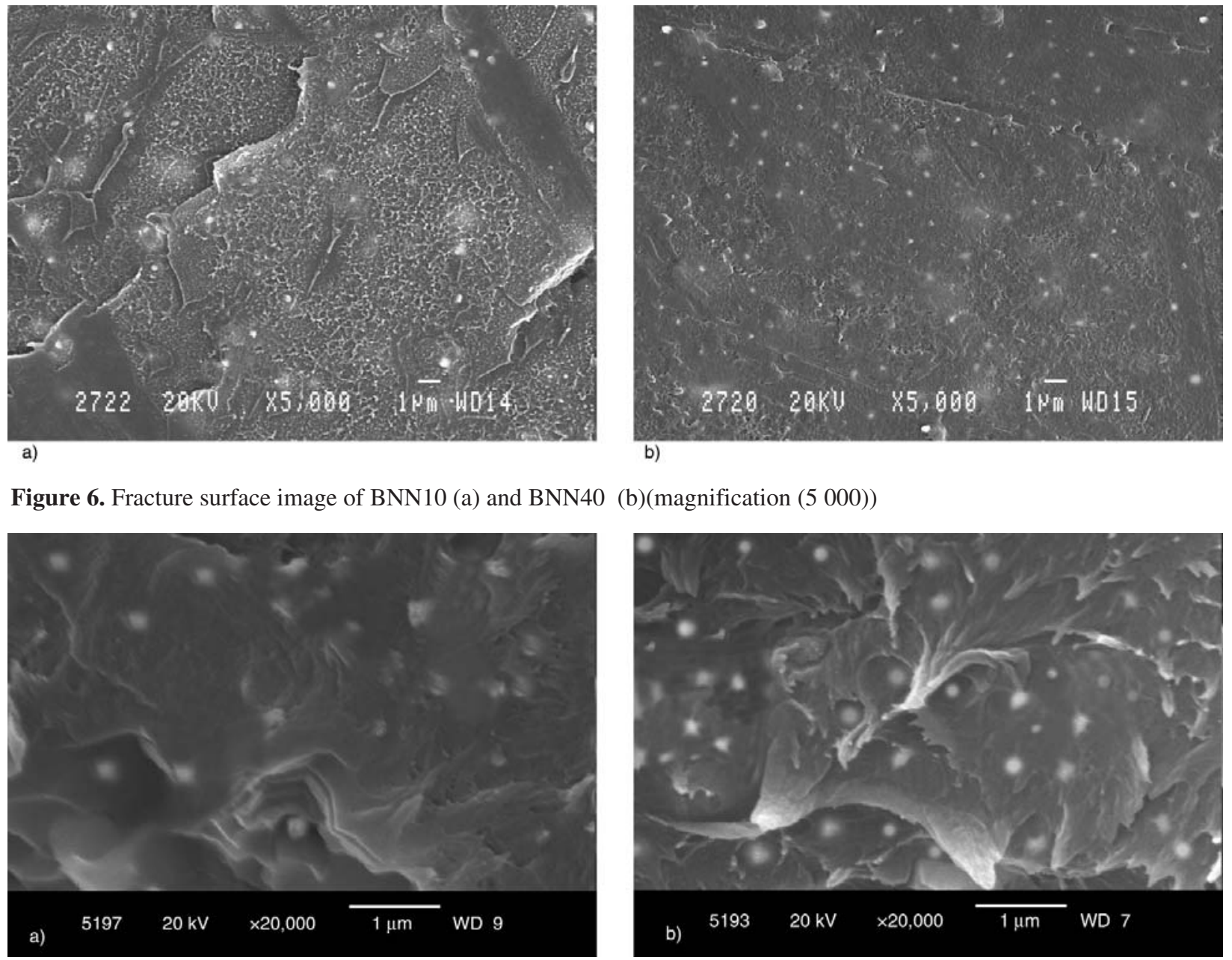

b)

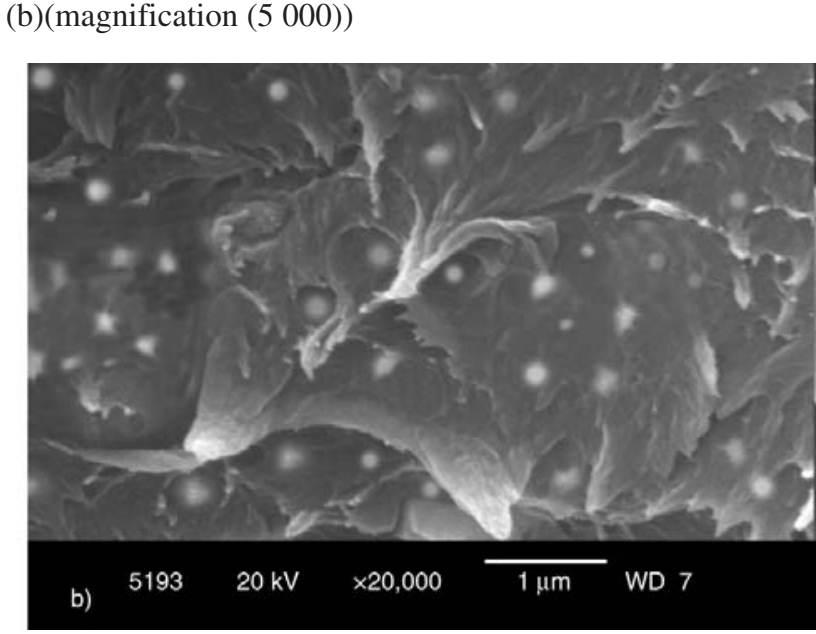

Figure 7. Fracture surface image of BNN10 (a) and BNN40 (b) (magnification (20 000))
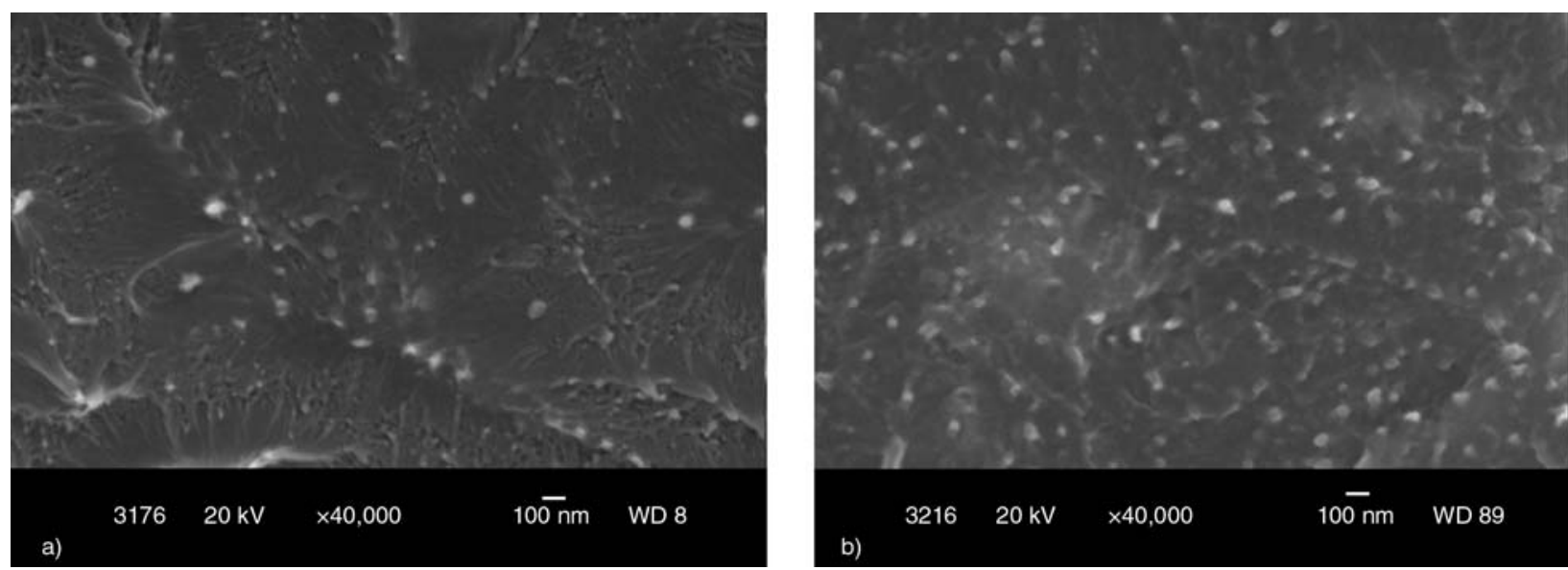

Figure 8. Fracture surface image of BNN10 (a) and BNN40 (b) (magnification (40 000))

PS composites, the active ceramic phase is not selfconnected and the passive polymer phase is connected by the three dimensions by maintaining the (0-3) connectivity. The particle dispersion and particle-matrix reinforcement play vital roles for both tensile and elongation properties of the composites. In all pictures it is seen that all the particles are neatly coated with PS. The ceramic particles are dispersed homogeneously with the interspaces filled with PS at all magnification level, and large defects are not observed. Figures $6-8 \mathrm{a}$ and $6-8 \mathrm{~b}$ showed a dense microstructure and is believed to have affected the mechanical properties of the composites. The size of the dispersed particle can be 
calculated by counting a large number of particles from various micrographs of different magnification and quantitatively analyzed in terms of their diameter [26].

The number average particle diameter is given by Equation (4):

$$
\frac{\sum N_{i} D_{i}}{N_{i}}=\bar{D}_{n}
$$

The weight average particle diameter is given by Equation (5):

$$
\frac{\sum N_{i} D_{i}^{2}}{\sum N_{i} D_{i}}=D_{w}
$$

The volume average particle diameter is given by Equation (6):

$$
\frac{\sum N_{i} D_{i}^{3}}{\sum N_{i} D_{i}^{2}}=D_{v}
$$

where $N_{i}$ is the number of particles having diameter $D_{i}$. The polydispersity index, a measure of particle size distribution and it is calculated as Equation (7):

$$
\frac{D_{w}}{D_{n}}=P D I
$$

The calculated polydispersity index is less than 1.2, reported in Table 5 and the value gives clear evidences for the good dispersion of filler in the matrix [27].

\subsubsection{Density calculations}

The first second and third column of Table 6 reports the density pattern of the prepared composites.

Table 5. Particle diameter measurement [nm]

\begin{tabular}{|c|c|c|c|c|}
\hline $\begin{array}{c}\text { Name of } \\
\text { sample }\end{array}$ & $\begin{array}{c}\text { Number } \\
\text { average }\end{array}$ & $\begin{array}{c}\text { Weight } \\
\text { average }\end{array}$ & $\begin{array}{c}\text { Volume } \\
\text { average }\end{array}$ & PDI \\
\hline BNN10 & 65.47 & 78.36 & 89.79 & 1.196 \\
\hline BNN40 & 52.50 & 61.25 & 84.96 & 1.166 \\
\hline
\end{tabular}

The theoretical density values are calculated using the rule of mixtures [28] (Equation (8)):

$$
\rho_{c}=\rho_{p}\left(1-v_{1}\right)+\rho_{f} v_{1}
$$

where $\rho_{c}$ is the density of the composite, $\rho_{p}$ is the density of phase 1 (polymer phase), $\rho_{f}$ is the density of phase 2(ceramic phase) and $v_{1}$ is the volume fraction of filler. The values of $\rho_{p}$ and $\rho_{f}$ are taken to be 1050 and $5950 \mathrm{~kg} \cdot \mathrm{m}^{-3}$ respectively. First and second column of Table 6 compares the average of the measured density values (by sample geometry and Archimedes methods) with theoretical values. As density measurements of BNN-PS, it is revealed that the composite pellets followed the rule of mixtures with respect to the constituent powders, so that no noticeable change in density is induced by the thermal processing [29].

\subsection{Mechanical properties}

\subsubsection{Stress Strain Behavior}

So many investigators discussed the mechanical property behavior of filled polymer systems. These reports reveal that the tensile modulus is the easiest property to estimate because it is a bulk property that depends primarily on the geometry, particle size distribution and concentration of the filler. The two principal parameters used to describe the mechanical behavior of polymers are stress and strain. In the initial stages of the stress, the strain increases linearly (Hookean region). Modulus is the ratio of the stress to strain in the linear region of the stress strain curve. It is well known that the modulus increases for a polymer when mineral filler is incorporated into it. The exact nature of the tensile response of a polymeric material depends upon the chemical structure of the polymer, conditions of the sample preparation, molecular weight, molecular weight distribution, and the extend of any cross linking or branching [30].

At normal temperature and pressure, polystyrene exhibits an increase in stress with increasing strain

Table 6. Some representative properties of the composites

\begin{tabular}{|c|c|c|c|c|}
\hline Name of the sample & $\begin{array}{c}\text { Theoretical density } \\
{\left[\mathbf{k g m}^{-3}\right]}\end{array}$ & $\begin{array}{c}\text { Observed density } \\
{\left[\mathbf{k g m}^{-3}\right]}\end{array}$ & $\begin{array}{c}\text { Ultimate stress } \\
{[\mathbf{M P a}]}\end{array}$ & $\begin{array}{c}\text { Young's modulus } \\
{[\mathbf{G P a}]}\end{array}$ \\
\hline Polystyrene & 1050 & 1050 & $36.61 \pm 0.5$ & $2.89 \pm 0.02$ \\
\hline BNN10 & 1542 & 1538 & $45.68 \pm 0.35$ & $3.60 \pm 0.04$ \\
\hline BNN20 & 2033 & 2030 & $54.75 \pm 0.45$ & $4.31 \pm 0.02$ \\
\hline BNN30 & 2524 & 2516 & $64.12 \pm 0.5$ & $5.18 \pm 0.03$ \\
\hline BNN40 & 3014 & 3010 & $72.15 \pm 0.35$ & $5.68 \pm 0.03$ \\
\hline
\end{tabular}




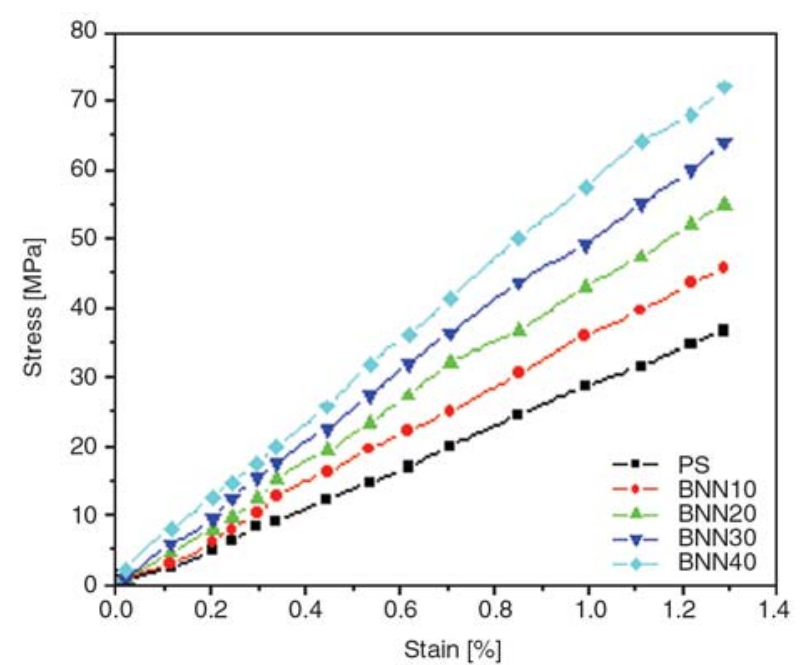

Figure 9. Stress strain behavior of polystyrene nanocomposites

up to the point of sample failure. The stress at failure is called ultimate stress or stress at break. Typical value of modulus and ultimate stress of the PS and composites are reported in the Table 6 .

A very good linear fit is obtained for all the curves in Figure 9, with $99.77 \%$ correctness by origin plot indicates a relatively homogeneous distribution of filler particles in the matrix; structure related phenomena (change of matrix properties by the introduction of the filler, much aggregation, and segregation of particles during processing) seem to be absent in the composites.

\subsubsection{Theoretical modeling}

The mechanical properties of two-phase composites made up of a continuous polymer phase and particulate filler phase have been studied in great detail. As a result a variety of models are available to describe the modulus, tensile strength, and elongation at break as a function of filler volume fraction. The modeling and simulation of polymerbased composites has become an important topic in recent times because of the need for the development of these materials for engineering applications.

The mechanical properties of particulate filled composites are affected by a number of parameters such as filler orientation, particle size of the filler and filler-matrix adhesion etc. The load transfer from matrix to filler in a composite is strongly related to optimum mechanical properties of the composites.

Several theories have been proposed to model the tensile modulus of 'the non-interactive' composite materials in terms of different parameters. Among the most prominent, historically and technically are those developed by Einstein [31, 32], with and without adhesion, Kerner [33], and Sato and Furukawa [34]. Modified Kerner equation is an upper bound one while considering adhesion between filler and polymer. Einstein and Sato and Furukawa models are calculated with various adhesion parameters.

\section{Einstein equation (with out adhesion parameter) (Equation (9)):}

$M_{c}=M_{m}\left(1+v_{1}\right)$

where $M_{c}$ and $M_{m}$ are the Young's modulus of the composite and the matrix respectively and $v_{1}$ the filler volume percentage.

\section{Einstein equation (with adhesion parameter) \\ (Equation (10)):}

$M_{c}=M_{m}\left(1+2.5 \cdot v_{1}\right)$

where the constant 2.5 is accounted for adhesion between filler and polymer.

\section{Kerner equation:}

Kerner equation can be used to estimate the modulus (Equation (11)):

$$
M_{c}=M_{m}\left[1+\frac{v_{1} 15(1-r)}{V_{m}(8-10 r)}\right]
$$

where $V_{m}$ is the matrix volume fraction and $r$ is the Poisson's ratio of the matrix. For expansible polymers incorporating rigid spherical particles featuring adhesion, the Kerner equation can be used to estimate the modulus.

\section{Sato and Furukawa:}

Sato and Furukawa have developed an expression (Equations (12) and (13)) for the modulus for the case where the adhesion is taken as a parameter: 
$M_{c}=M_{m}\left[\left(1+\frac{v_{1}^{2 / 3}}{2-2 v_{1}^{1 / 3}}\right)(1-\psi j)-\frac{v_{1}^{2 / 3} \psi j}{\left(1-v_{1}^{1 / 3}\right) v_{1}}\right]$

$\psi=\left(\frac{v_{1}}{3}\right) \frac{1+v_{1}^{1 / 3}-v_{1}^{2 / 3}}{1-v_{1}^{1 / 3}+v_{1}^{2 / 3}}$

where $v_{1}$ is the volume fraction of filler $j$ is the adhesion parameter, $j=1$ for poor adhesion, and $j=0$ for perfect adhesion. It can be seen (from Figure 10) that the experimental results are placed in between Kerner and Sato Furukawa relations with perfect adhesion.

All these predictions assume that the matrix and filler have an appreciable degree of physical interaction only. The polymer matrix is stiffened by the particulate filler. In most conventionally filled polymer systems the modulus increases linearly with the filler volume fraction. The enhancement of the modulus for BNN-PS may not be attributed simply by the introduction of the high modulus inorganic filler to PS matrix.

The increase of modulus is mainly governed by the particle size of the filler [35]. With Sato and Furukawa relations Young's modulus can be calculated with different adhesion parameters such as the value of adhesion parameter $j=1$ for poor adhesion, $j=0.5$ for medium adhesion and $j=0$ for good adhesion. The result obtained with adhesion parameter $(j=0)$ is closely fitted with the experimental curve evidenced that this type of perfect adhesion is the cause of increment in both Young's

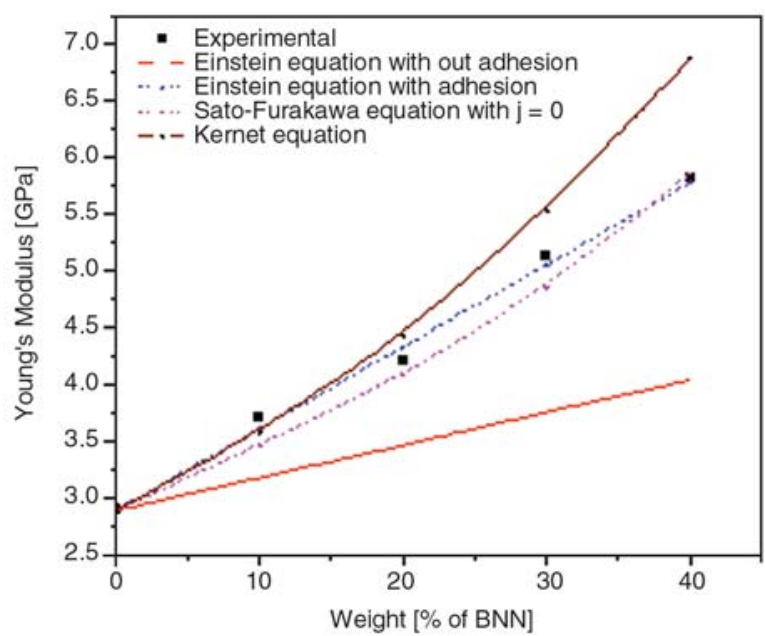

Figure 10. Theoretical modeling of the tensile modulus of BNN-PS composites with different adhesion parameters modulus and tensile strength of the composites as in Figure 10.

\subsubsection{Tensile strength}

The tensile strength of a filled polymer is more difficult to predict because it depends strongly on local polymer filler interactions. Tensile strength is the force required to pull the composite to the point where it breaks. Specifically the tensile strength of a material is the maximum amount of tensile stress that it can be subjected to before failure. Brittle materials, such as PS, do not have a yield point, which means that ultimate strength and breaking strength are same.

The effect of volume fraction of BNN on tensile strength of the composites is given in Table 6 . The virgin PS shows a tensile strength of around $36 \mathrm{MPa}$, coincides with its reported values [36]. Tensile strength of the composites increases with BNN filler loading. One problem in particulatefilled composites is the poor stress transfer at the filler-polymer interface because of the non-adherence of the filler to the polymer. The nanocomposites showed an increment in its ultimate stress value. In heterogeneous polymer systems the mechanism of micromechanical deformations and consequently, the macroscopic properties of the polymers are determined by local stress distribution around the inclusions. Because the adherence of filler to polymer in BNN-PS composites, the filler particles are able to carry any load, making it a strong body. Stress distribution will be created around the particles, increasing the composite strength further.

\subsubsection{Theoretical modeling of tensile stress}

A simple model was used by Lubin [37] for the determination of tensile stresses in unfilled and filled polymer with inclusions. It is assumed that at the composite tensile strength (ultimate tensile stress), the polymer has undergone maximum plastic deformation. Moreover, the load carried by the components corresponds to their effective crosssections occupied in the specimen, i.e. $\left(1-1.21 v_{1}^{2 / 3}\right)$ for the matrix and $\left(1.21 v_{1}^{2 / 3}\right)$ for the inclusions, where $v_{1}$ is the volume fraction of inclusions in the composites [38]. If it is assumed that the average 
stress acting across the surface of the fillers is $£^{*}$, the Equation (14) must be valid:

$£^{*}=£_{m}\left(1-1.21 v_{1}^{2 / 3}\right)+£^{*}\left(1.21 v_{1}^{2 / 3}\right)$

where $£$ and $£_{m}$ are the composite and matrix tensile stresses, respectively. The load carried by the filler $\left(£^{*}\right)$ is always very much smaller than the matrix tensile stress when the filler with large particle size is used, i.e. debounding takes place. When the specific surface area of the filler is larger (smaller particles), $£^{*}$ can significantly exceed the tensile strength of the matrix. Considering the composition-dependence of tensile stress of composites including adhesion parameter Pukánszky [39] arrived the following relation (Equation (15)):

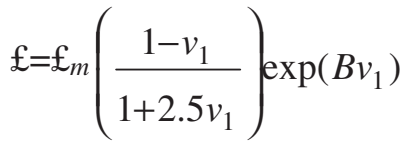

where $B$ is an empirical parameter characterizing the degree of particle matrix reinforcement. It increases with specific surface area and the adhesive strength of the filler polymer interphase. The value of the parameter is found out by plotting tensile strength of the composites with filler volume fraction and linear fitting the experimental data as in Figure 11. The value of $B$ is 4.69. An increased modulus is a well known fact in particulate filled micro composites. But an increment in tensile modulus associated with an increment in tensile strength is reported as the peculiarity of the nano composites [40]. These results show that the small BNN particles enable both relatively good disper-

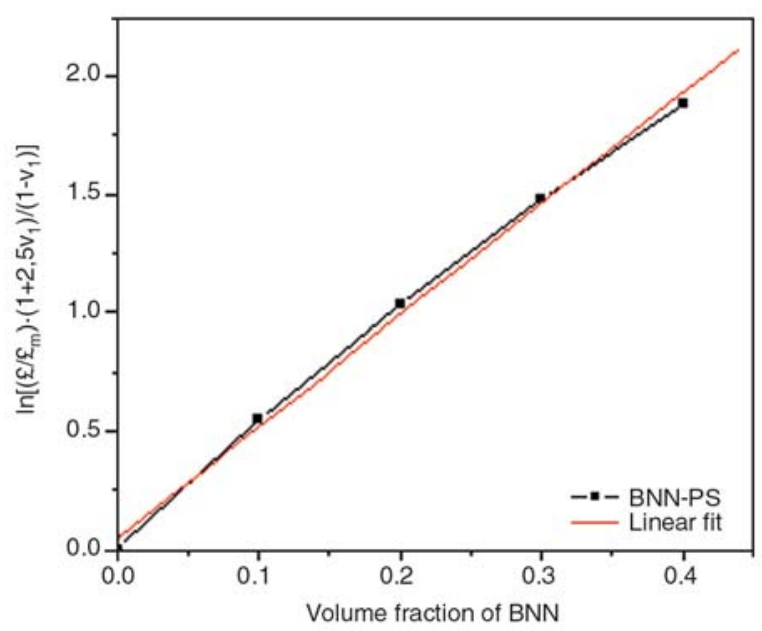

Figure 11. Adhesion parameter from the tensile stress of BNN-PS composites sion and improved interfacial stress transfer in BNN-PS composites. In contrast with the tensile modulus, theoretical predictions of the tensile strength are less highly developed.

\subsubsection{Elongation at break}

Normally addition of rigid particulate fillers to a polymer matrix decreases the elongation at break (strain at break). Only in rare instances, if there is a good reinforcement between polymer and the filler, the fracture goes from particle to particle rather than following a direct path, and these filled polymers have nearly equal elongations at break when compared with neat polymer. BNN-PS composites show the trend of perfect adhesion by the elongation at break value. Elongation at break value of BNN-PS composites is almost same as that of neat PS showing the good reinforcement between the filler and the matrix. A basic model that describes the elongation at break given by Equation (16) [41-43]:

$€=€_{m}\left(1-v_{1}^{1 / 3}\right)$

where $€$ and $€_{m}$ are the elongations at break of the composites and the unfilled polymer, respectively. For the case of perfect adhesion, under the assumption that the polymer breaks at the same elongation in the filled system as in the neat polymer. By Equation (15) it is believed that in the case of poor adhesion, the elongation is expected to decrease more gradually with filler loading.

\section{Conclusions}

Polycrystalline BNN ceramic powder with particle size less than $100 \mathrm{~nm}$ has been synthesized using conventional ceramic technique. The morphology and tensile behavior of BNN-PS composites are studied in detail. The most fascinating property of the composites is the relatively good dispersion of the filler particles in the matrix. The particle dispersion and particle-matrix reinforcement play vital roles for both tensile and elongation properties of the composites. Young's modulus and tensile strength of polystyrene show an increment by the incorporation of BNN filler. Elongation at break value of the composites is same as that of neat PS showing the good reinforcement between the filler and the matrix and stress transfer at the filler-poly- 
mer interface is effectively possible by the efficient dispersion and good adherence of the filler to polymer.

\section{References}

[1] Byrene M. T., McNamee W. P., Gun'ko Y. K.: Chemical functionalization of carbon nanotubes for the mechanical reinforcement of polystyrene composites. Nanotechnology, 19, 415707-415714 (2008). DOI: $10.1088 / 0957-4484 / 19 / 41 / 415707$

[2] Jancar J., Kucera J.: Yield behaviour of polypropylene filled with $\mathrm{CaCO}_{3}$ and $\mathrm{Mg}(\mathrm{OH})_{2}$ 'zero' interfacial adhesion. Polymer Engineering and Science, 30, 707711 (1990)

DOI: $10.1002 /$ pen.760301203

[3] Yoon C-B., Lee S-H., Lee S-H., Koh Y-H., Kim H-E., Lee K-W.: Piezoelectric multilayer ceramic/ polymer composite transducer with 2-2 connectivity. Journal of the American Ceramic Society, 89, 2509-2513 (2006).

DOI: 10.1111/j.1551-2916.2006.01080.x

[4] Psarras G. C.: Nanodielectrics: An emerging sector of polymer nanocomposites. Express Polymer Letters, 2, 460 (2008).

DOI: $10.3144 /$ expresspolymlett.2008.55

[5] Dekkers M. E., Heikens D.: The effect of interfacial adhesion on the tensile behavior of polystyrene-glassbead composites. Journal of Applied Polymer Science, 28, 3809-3815 (1983). DOI: 10.1002/app.1983.070281220

[6] Smay J. E., Cesarano S., Tuttle B. A., Lewis J. A.: Piezo electric properties of 3-X periodic $\mathrm{Pb}\left(\mathrm{Zr}_{\mathrm{X}} \mathrm{Ti}_{1-\mathrm{x}}\right) \mathrm{O}_{3^{-}}$ polymer composites. Journal of Applied Physics, 92, 6119-6127 (2002).

DOI: $10.1063 / 1.1513202$

[7] Pradhan D. K., Choudhary R. N. P., Samantaray B. K.: Studies of structural, thermal and electrical behavior of polymer nanocomposite electrolytes. Express Polymer Letters, 2, 630-638 (2008).

DOI: $10.3144 /$ expresspolymlett.2008.76

[8] Tjong S. C.: Structural and mechanical properties of polymer nanocomposites. Material Science and Engineering: Reports, 53, 73-197 (2006). DOI: $10.1016 /$ j.mser.2006.06.001

[9] Nanda M., Tripathy D. K.: Physico-mechanical and electrical properties of conductive carbon black reinforced chlorosulfonated polyethylene vulcanizates. Express Polymer Letters, 2, 855-865 (2008). DOI: $10.3144 /$ expresspolymlett.2008.100

[10] Hameed N., Thomas S. P., Abraham R., Thomas S.: Morphology and contact angle studies of poly(styreneco-acrylonitrile) modified epoxy resin blends and their glass fibre reinforced composites. Express Polymer Letters, 1, 345-355 (2007). DOI: $10.3144 /$ expresspolymlett.2007.49
[11] Panda A. B., Pathak A., Pramanik P.: Low temperature preparation of nanocrystalline solid solution of strontium-barium-niobate by chemical process. Material Letters, 52, 180-186 (2002). DOI: $10.1016 / \mathrm{S} 0167-577 \mathrm{X}(01) 00389-5$

[12] Thakur A. K., Prahan D. K., Samantaray B. K., Choudhary R. N. P.: Studies on an ionically conducting polymer nanocomposite. Journal of Power Sources, 159, 272-276 (2006). DOI: 10.1016/j.jpowsour.2006.04.096

[13] Jamieson P. B., Abraham S. C., Bernstein J. L.: Ferroelectric tungsten bronz-type crystal structures I. Barium sodium niobate. Journal of Chemical Physics, 48 , 5048-5060 (1968).

DOI: $\underline{10.1063 / 1.1668176}$

[14] Gaudet J., Taveres A. C., Transati S., Guay D.: Physicochemical characterization of mixed $\mathrm{RnO}_{2}$ $\mathrm{SnO}_{2}$ solid solutions. Chemistry of Materials, 17, 1570-1579 (2005).

DOI: $\underline{10.1021 / \mathrm{cm} 0481291}$

[15] Bruneel E., Persyn F., Hoste.: Mechanical and superconducting properties of $\mathrm{BiPbSrCaCuO}-\mathrm{PE}$ and $\mathrm{BiPbSrCaCuO}-\mathrm{MgO}$ composites. Superconductor Science and Technology, 11, 88-93 (1998). DOI: $\underline{10.1088 / 0953-2048 / 11 / 1 / 018}$

[16] Vollenberg P. H. T., Heikens D.: Particle size dependence of the Young's modulus of filled polymers: 1. Preliminary experiments. Polymer, 30, 1656-1665 (1989). DOI: 10.1016/0032-3861(89)90326-1

[17] Saq'an S. A., Ayesh A. S., Zihlif A. M., Martuscelli E., Ragosta G.: Physical properties of polystyrenealum composites. Polymer Testing, 23, 739-745 (2004).

DOI: $10.1016 /$ j.polymertesting.2004.04.008

[18] Sharatsingh N. K., Sharma N.: X-ray - A boon for elemental analysis. Resonance, 10, 60-69 (2005). DOI: $10.1007 / \mathrm{BF} 02895795$

[19] Calligaris M., Silvanio G.: Powder diffraction package verson 1.1. in 'Proceedings of third international school and workshop of rystallography on X-ray powder diffraction and its applications' Cairo, Egypt, 456470 (1990).

[20] Zuo R., Granzow T., Lupascu D. C., Rödel J.: PMNPT ceramics prepared by spark plasma sintering. Journal of the American Ceramic Society, 90, 1101-1106 (2007).

[21] Warren B. E.: X-ray diffraction in random layer lattice. Physical Review, 59, 693-698 (1941). DOI: $\underline{\text { 10.1103/PhysRev.59.693 }}$

[22] Mori S., Yamamoto N., Koyama Y., Uesu Y.: Evolution of a ferroelastic domain structure in an incommensurate phase of barium sodium niobate ( $\mathrm{Ba}_{2} \mathrm{NaNb}_{5} \mathrm{O}_{15}$ ). Physical Review B, 52, 6158-6165 (1995).

DOI: 10.1103/PhysRevB.52.6158 
[23] Panajkar M. S., Phatak G. M., Gangadharan K., Gopalakrishnan I. K., Gopinathan C.: Preparation of high Tc-superconducting ceramic/polymer composites using gamma radiation. Journal of Material Science Letters, 16, 218-220 (1997).

[24] Lee W. E., Arshad S. E., James P. F.: Importance of crystallization hierarchies in microstructural evolution of silicate glass-ceramics. Journal of American Ceramic Society, 90, 727-737 (2007). DOI: $10.1111 / j .1551-2916.2006 .01479 . x$

[25] Newnham R. E.: Structure - Property relations. Springer-Verlag, Berlin (1975).

[26] Bliznakov E. D., White C. C., Shaw M. T.: Mechanical properties of blends of HDPE and recycled ureaformaldehyde resin. Journal of Applied Polymer Science, 77, 3220-3227 (2000).

DOI: $10.1002 / 1097-4628(20000929) 77: 14<3220::$ AIDAPP250>3.0.CO;2-4

[27] Ray D. A.: On the modulus of particulate filled composites: Application of van der Poel's equation. Journal of Polymer Science Polymer Physics, 14, 2073$2082(2003)$ DOI: $10.1002 /$ pol.1976.180141112

[28] Satish B., Sridevi K., Vijaya M. S.: Study of piezoelectric and dielectric properties of ferroelectric PZTpolymer composites prepared by hot-press technique. Journal of Physics D: Applied Physics, 35, 2048-2050 (2002).

DOI: $10.1088 / 0022-3727 / 35 / 16 / 321$

[29] Huang Q-W., Wang P-L., Cheng Y-B., Yan D-S.: $\mathrm{XRD}$ analysis of formation of strontium barium niobate phase. Materials Letters, 56, 915-920 (2002). DOI: $\underline{10.1016 / \mathrm{S} 0167-577 X(02) 00637-7}$

[30] Prashantha K., Soulestin J., Lacrampe M. F., Claes M., Dupin G., Krawczak P.: Multi-walled carbon nanotube filled polypropylene nanocomposites based on masterbatch route: Improvement of dispersion and mechanical properties. Express Polymer Letters, 2, 735-745 (2008).

DOI: $10.3144 /$ expresspolymlett.2008.87

[31] Cowie J. M. G.: Polymers: Chemistry and physics of modern materials. Nelson Thornes Ltd., Cheltenham (2000).
[32] Nielsen L. E., Landel R. F.: Mechanical properties of polymers and composites. Hanser Publishers, New York (1992).

[33] Selvin T. P., Kuruvilla J., Sabu T.: Mechanical properties of titanium dioxide filled polystyrene microcomposites. Material Letters, 58, 281-289 (2004). DOI: $\underline{10.1016 / \mathrm{S} 0167-577 \mathrm{X}(03) 00470-1}$

[34] Ehrig R.: Plastic recycling. Hanser, New York (1992).

[35] van Krevelan D. W.: Properties of polymers: Their correlation with chemical structure. Elsevier, Amsterdam (1972).

[36] Joel R .F.: Polymer Science and Technology. PrenticeHall of India, New Delhi (2000).

[37] Lubin G.: Handbook of composites. van Nostrand Reinhold, London (1982).

[38] Shang S. W., Williams J. W., Söderholm K-J. M.: Using the bond energy density to predict the reinforcing ability of a composite. Materials Science, 27, 4949-4956 (1992). DOI: $10.1007 / \mathrm{BF} 01105259$

[39] Pukánszky B.: Particulate filled polypropylene: Structure and properties. in 'Polypropylene: Structure, blends and composites' (ed. Karger-Kocsis J.) Chapman and Hall, London, Vol 3, 1-70 (1995).

[40] Vollenburg P. H. T., de Haan J. W., van de Ven L. J. M., Heikens D.: Particle size dependence of the Young's modulus of filled polymers: 2 . Annealing and solid-state nuclear magnetic resonance experiments. Polymer, 30, 1663-1665 (1989). DOI: $10.1016 / 0032-3861(89) 90327-3$

[41] Nielsen L. E., Landel R. F.: Mechanical properties of polymers and composites. Marcel Dekker, New York (1994).

[42] Thomas S. P., Thomas S., Abraham R., Bandyopadhyay S.: Polystyrene/calcium phosphate nanocomposites: Contact angle studies based on water and methylene iodide. Express Polymer Letters, 2, 528538 (2008).

DOI: $10.3144 /$ expresspolymlett.2008.63

[43] Luo J-C., Wen H-C., Wuz W-F., Chou C-P.: Mechanical research of carbon nanotube/PMMA- composite films. Polymer Composites, 29, 1285-1290 (2008). DOI: $\underline{10.1002 / p c .20388}$ 